\title{
Objective Assessment of Tendinopathy by Ultrasound Elastography
}

\section{Rajul Rastogi ${ }^{1 *}$ Neha $^{2}$, Vijai Pratap ${ }^{3}$ and GL Meena ${ }^{4}$}

${ }^{1}$ Associate Professor, Department of Radiodiagnosis, Teerthanker Mahaveer Medical College and Research Center, Moradabad, Uttar Pradesh, India

${ }^{2} P G$ Resident, Department of Radiodiagnosis, Teerthanker Mahaveer Medical College and Research Center, Moradabad, Uttar Pradesh, India

${ }^{3}$ Professor, Department of Radiodiagnosis, Teerthanker Mahaveer Medical College

and Research Center, Moradabad, Uttar Pradesh, India

${ }^{4}$ Professor, Department of Radiodiagnosis, S.N. Medical College and Hospital, Jodhpur,

Rajasthan, India

*Corresponding Author: Rajul Rastogi, Associate Professor, Department of Radiodiagnosis,

Teerthanker Mahaveer Medical College and Research Center, Moradabad, Uttar Pradesh, India.

DOI: 10.31080/ASOR.2020.03.0189

\begin{abstract}
Tendinopathy refers to an inflammatory condition of tendon usually secondary to overuse. In the tendons, edema leads to internal softening with consequent decrease in echogenicity and elasticity hence the tendon will appear hypoechoic on B-mode, gray-scale ultrasonography while appearing brighter on ultrasound elastography than its normal counterpart tendon.

Ultrasound Elastography is a recent noninvasive technique that allows evaluation of the elasticity of human tissues. Acoustic radiation forced impulse is a special form of ultrasound elastography wherein an acoustic radiation impulse is sent to the human tissues and the strength of returning echo indicates the elasticity of tissues. The latter can be depicted in shades of gray or different colors. If the image is coded with shades of gray, harder tissues will appear black while softer tissues will show varying shades of gray. However, in color-coded maps red or blue is indicated for hard tissue and blue or red respectively indicating soft tissue.

Majority of the studies in the recent literature focusses on ultrasound elastography of evaluation of larger tendons especially the Achilles tendon, rotator cuff, quadriceps tendon, etc. while very few studies have focused on smaller tendons of the human body. Hence, this study primarily focusses on objectively evaluating the role of ultrasound elastography in assessment of tendinopathy at wrist joint.
\end{abstract}

Keywords: Ultrasound Elastography; Tendinopathy; Wrist Joint; Tenomalacia

\section{Introduction}

Excessive use results in varying degrees of inflammation of tendon referred to as tendinopathy [1]. Tendinopathy is the commonest form of musculoskeletal injury and the upper limb tendinopathy carries a significant financial impact for an individual by affecting their working capacity [2]. Early diagnosis of tendinopathy is also needed to avoid severe tendinous injuries including tendon rupture [3].

Whatsoever the cause, high-resolution, B-mode, ultrasonography (HRUS) is the modality of choice for evaluation in tendinopathy as it has excellent clinical and histopathological correlation [1]. Varying degrees of inflammation of tendon leads to variable alterations in thickness and edema with subsequent reduction in echogenicity, well-detected on HRUS.

However, in certain cases where the individual with tendinopathy is symptomatic, the variations in thickness and echogenicity of affected tendon are subtle enough to escape detection on HRUS. In such cases, the softening of tendon termed tenomalacia that results in reduced elasticity can be utilized to make an early diagnosis allowing early institution of management [4]. This alteration in tendon elasticity can be assessed objectively by ultrasound elastography (USE), a recent added dimension to HRUS [1-3].

USE is based on the assessment of intrinsic internal displacement within human tissues following application of extrinsic force which is directly proportional to the elastic properties of the tissues [3]. USE can be compressive-strain and shear-wave types. The former is more subjective as it is dependent on the external compressive force applied by the examiner while shear-wave elastography is independent of external compression and relies on the calculations made by the ultrasound-system on the basis of shearwave applied and generated providing more objective and reliable results $[1,3]$. 
Hence, in tendinopathy, the thickness of tendon may be increased, normal or reduced but its firmness and elasticity is reduced i.e. tendon shows tenomalacic changes resulting in brighter (gray shade instead of black) tendon on SWE [1-4].

\section{Aim of the Study}

To determine the usefulness of Ultrasound elastography (USE) in early detection of tendinopathy in small tendons at wrist joint.

\section{Materials and Methods}

Four patients with clinical diagnosis of unilateral tendinopathy at wrist joint but with normal high-resolution, B-mode ultrasound were included in the study. All the examinations were performed by very-high frequency, linear-array transducer (14 - $18 \mathrm{MHz})$ using ARFI on Siemens Acuson S2000 Ultrasound scanner. PostB-mode scanning, ultrasound elastography was performed using virtual tissue imaging and the generated elastogram was recorded in form of gray-scale coded maps.

Patients with history of trauma, inflammatory arthropathy, previous surgery and pregnancy were excluded from the study.

\section{Observations and Analysis}

Two patients with suspected unilateral tendinopathy of rightsided flexor carpi ulnaris that did not show significant findings on B-mode, HRUS (Figure 1a) revealed significantly reduced elasticity with tendon appearing brighter/gray (Figure 1b) on ARFI-based elastogram than its asymptomatic contralateral counterpart tendon (Figure 1c).

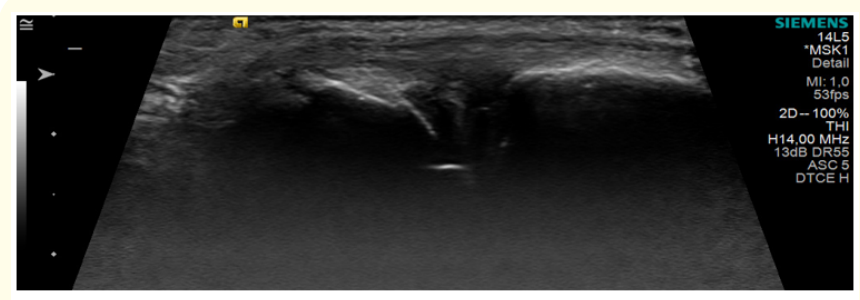

Figure 1a

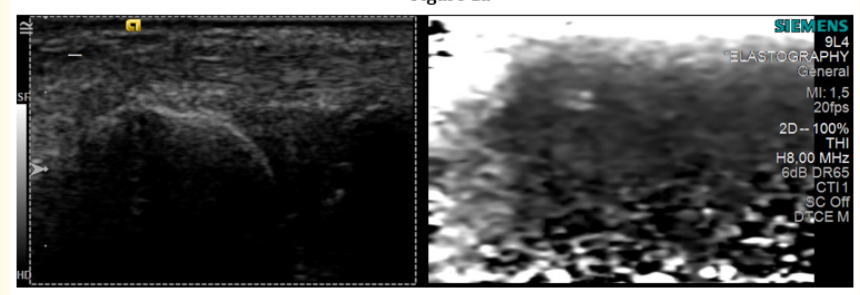

Figure 1b

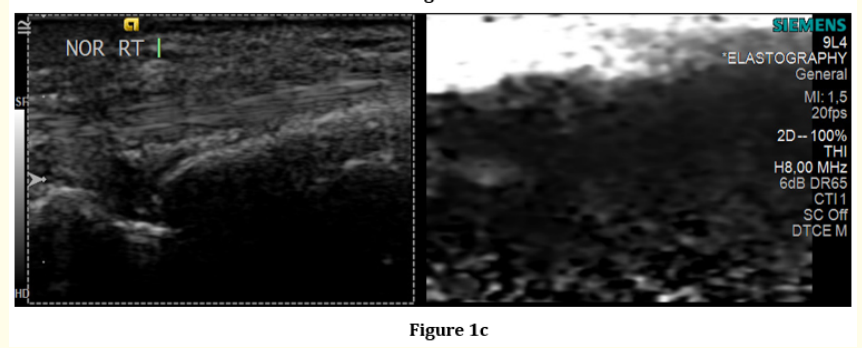

In another two patients with suspected unilateral tendinosis of abductor pollicis longus B-mode, HRUS revealed only thickened synovium (Figure 2a) but the ARFI-based elastogram revealed significantly reduced elasticity with tendon appearing gray but less brighter than softer thickened synovium (Figure $2 \mathrm{~b}$ ).

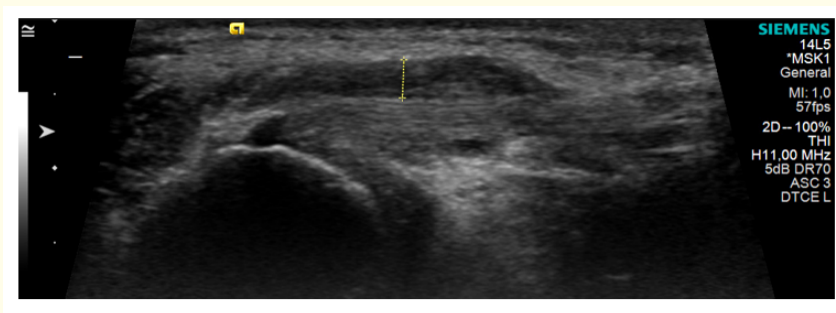

Figure 2a

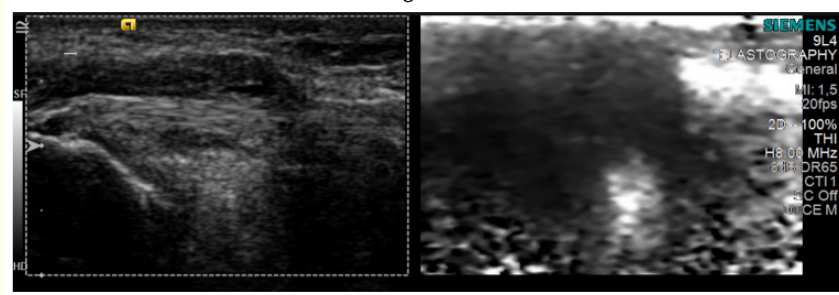

Figure 2b

Figure 2

The above-described findings in all four patients reveal that ARFI-based USE is useful in early objective detection of tendinopathy in symptomatic tendons with normal B-mode, HRUS.

\section{Discussion}

Recently, there has been a growing interest on evaluating tendinopathy by USE due to its ability of early objective detection [1]. Nearly all tendons are amenable to USE but evaluation of smaller tendons is still challenging due to technical limitations. USE is a reliable technique for tendinopathy providing higher sensitivity, specificity and accuracy over HRUS [1-3]. Whether compressive-strain (CSE) or shear-wave (SWE) elastography is used, but they are found superior to B-mode HRUS in all symptomatic tendinopathy patients by all previous studies $[1,2]$.

USE is not only a non-invasive, cost and time-effective and reliable tool for assessing tendinopathy but with its added dynamicity and contralateral comparison makes it the screening and diagnostic imaging tool in majority of cases prior to any advanced imaging like magnetic resonance imaging [1].

Majority of the studies on the USE evaluation of tendinopathy have been performed on large tendon with very fewer studies like our study have been conducted on objective evaluation of tendinopathy involving small tendons like those at wrist joint by USE [13,5-9]. Irrespective of the tendon evaluated and type of USE utilised in the study, tendinopathy is always associated with heterogeneous

Figure 1 
softening of tendon (tenomalacia) and reduced stiffness relative to its normal counterpart [4-6].

Despite several advantages, USE especially CWE is not free of limitations. CWE is not quantitative and is limited by subjective errors in applying external compressive forces [5,6]. Although SWE is quantitative yet it is limited by the minimum distance requirement ( $1-2 \mathrm{~mm}$ ) between the skin surface and region of interest and also it is not available with very-high frequency transducers used for optimal evaluation of small tendons especially of wrist and digits [5,6]. While acquiring USE, anisotropy must be avoided as artefacts in B-mode HRUS images affects its acquisition [6]. Also, while comparing the two elastogram, their size should be taken into consideration as the mean elasticity of all tissues within the elastogram is used to define the relative elasticity of tissue within the elastogram $[5,6]$.

Hence, our study though comprising of limited sample is unique in adding the further dimension of USE in small tendons of human body especially at the wrist joint. A study with higher number of patients may however be required to further validate the results of our study.

The further dimension of this study to objectively identify the reversal of the USE findings with relief in symptoms to develop an objective method of cessation of the therapy.

\section{Conclusion}

Ultrasound elastography can be used as an objective imaging tool for early detection of tendinopathy prior to any detectable alterations in thickness or echogenicity of tendons on B-mode, highresolution ultrasonography including the small tendons at wrist joint thus helping in early management and preventing further complications including tendon rupture. Ultrasound elastography may suffice in most cases as majority of tendons are amenable to USE except in certain cases where an advanced imaging modality like magnetic resonance imaging may be utilized.

\section{Bibliography}

1. Afandi R and Astawa P. "The Use of Elastography-Ultrasound in Diagnosing Tendinopathy Related Sport Injury: A 10 Years trend Systematic Review". The Orthopaedic Journal of Sports Medicine 7.11 (2019).

2. Washburn N., et al. "Ultrasound elastography and ultrasound tissue characterisation for tendon evaluation". Journal of Orthopaedic Translation 15 (2018): 9-20.

3. Prado-Costa R., et al. "Ultrasound elastography: compression elastography and shear-wave elastography in the assessment of tendon injury". Insights into Imaging 9.5 (2018): 791-814.
4. Khoury V and Cardinal E. “'Tenomalacia”: a new sonographic sign of tendinopathy?" European Radiology 19.1 (2009): 144146.

5. Kocyigit F., et al. "Investigation of biomechanical characteristics of intact supraspinatus tendons in subacromial impingement syndrome: a cross-sectional study with real-time Sonoelastography". American Journal of Physical Medicine and Rehabilitation 95.8 (2016): 588-596.

6. Elena Drakonaki. "Ultrasound elastography for imaging tendons and muscles". Journal of Ultrasonography 12.49 (2012): 214-225.

7. Buck AR., et al. "Detection of small tendon lesions by sonoelastographic visualization of strain profile differences: initial experiences". Skeletal Radiology 41.9 (2012): 1073-1079.

8. Lalitha P., et al. "Musculoskeletal applications of elastography: a pictorial essay of our initial experience". Korean Journal of Radiology 12.3 (2011): 365-375.

9. Klauser AS., et al. "Sonoelastography: Musculoskeletal applications”. Radiology 272.3 (2014): 622-633. 
The current standard of treatment for shaft femur fractures in adults is Closed Intramedullary Nailing [1,2]. Although union rates are reported to be $85-100 \%$, recent studies have shown high rate of Non-Union after Intramedullary Nailing [3]. The management of such cases still represents a challenge to the operating surgeon. Many risk factors for Aseptic Non-union of shaft femur have been identified viz. smaller undreamed nail, open fractures, tobacco consumption [1]. However, the rate of non-union of Distal Third fractures is higher due to inability to provide stable fixation in the distal fragment. This is due to the comparatively larger medullary cavity in the Distal Third fragment as compared to the isthmus thereby invariably using a smaller than warranted nail diameter

\begin{tabular}{|l|c|}
\hline \multicolumn{1}{|c|}{ Parameters } & Values \\
\hline Age (years), Mean & $32.70(16-64)$ \\
\hline Male/Female & $48 / 2$ \\
\hline Anatomical Classification & \\
\hline Isthmus & 29 \\
\hline Distal Third & 21 \\
\hline AO Classification & \\
\hline Type A & $43(86 \%)$ \\
\hline Type B & $7(14 \%)$ \\
\hline Type C & 0 \\
\hline Non-union type & \\
\hline Hypertrophic & $33(66 \%)$ \\
\hline Oligotrophic & $9(18 \%)$ \\
\hline Atrophic & $8(16 \%)$ \\
\hline
\end{tabular}

Table 1: Summary of characteristics of 50 patients with aseptic non-union of femur shaft.

$[4,5]$

According to current literature, exchange nailing remains the treatment of choice for aseptic non-union of femoral shaft [6]. This includes removal of previously inserted nail, over-reaming of medullary cavity by $2 \mathrm{~mm}$ and replacing with a larger diameter nail. Bone grafting is not required due to increased vascularity of nonunion site due to changes in the vascular dynamics at the fracture site [7]. Reaming increases the periosteal blood flow in response to endosteal circulation [8-10] thus stimulating bone formation at non-union site. In addition, there is mechanical stability offered by larger diameter nail and increased cortical contact due to reaming $[11,12]$.

However, conflicting data has emerged in the recent past for the management of Distal Third non-union of shaft femur. Exchange nailing alone has resulted in higher failure rate [5]. Furthermore, 2 studies questioned the effectiveness and outcomes of exchange nailing of isthmus non-union and demonstrated failure rate of
$27 \%$ [13] and $42 \%$ [14] respectively.

To obtain a clear consensus, this study was conducted: (1) To determine the functional and radiological outcome of reamed exchange nailing of isthmus aseptic non-union of shaft femur. (2) To determine if enhanced stability of distal fragment attained by placing blocking screws (poller) and distal interlocking screws in multiple planes gave acceptable union rates, radiological and functional outcomes.

\section{Materials and Methods}

Between 2006 and 2014, 55 patients with Aseptic Femoral Shaft Non-Union were analysed and treated at our institute. 5 patients were excluded from the study due to loss of patient follow up. Out of the remaining 50 patients included in our study, 29 were cases of Non-union of Isthmus and 21 were cases of Non-union of Distal Third region. All the patients included were cases previously operated with an intramedullary nail for a femoral shaft fracture and subsequently developed Non-union. All patients on presentation had a retained intramedullary nail. Nonunion for these patients was defined according to literature as missing union in treated fractures without progression towards healing. These patients in opinion of the treating surgeon had very little possibility of clinical and radiological union without any surgical intervention [15]. All patients presented with persistent pain, inability to bear weight and loss of function. Radiologically, lack of cortical bridging in 3 out of 4 cortices assessed in Antero-Posterior and Lateral conventional radiographs was considered confirmatory. None of the patients presenting in our OPD had any clinical or laboratory signs of infection nor any previous history during earlier treatment.

All patients with infected Non-unions of shaft femur, segmental defects more than $50 \%$ of cortical contact area, periprosthetic fractures, insufficiency fractures or pathological fractures were excluded from this study.

Out of the 50 patients included in this study, 48 were men and 2 women with a mean age of 32.70 (range 16 - 64 years) on presentation. Anatomically, 29 were non-union of Isthmus region and 21 were Non-union of Infra isthmus region. The mean duration of Non-union (Interval between first surgery and subsequent exchange nailing) was 11.60 months (range 7 - 25 months).

All patients were tested for infection preoperatively with complete blood count, ESR and CRP. No patient had any clinical or laboratory evidence of infection. Pre-operative templating was done with true size X-rays for all patients in standard AP and Lateral views. All surgeries were performed by the senior author at an Or- 
thopaedic Trauma Centre. All patients were followed up till union was achieved or until patients refused further management.

Healing was determined by the presence of bone bridging in 3 out of 4 cortices [16] on serially taken post-operative x-rays (Figure 1).

The surgical strategy adopted for isthmus nonunion was: (a) Removal of previous nail. (b) Over reaming by at least $2 \mathrm{~mm}$ followed by Exchange nailing. For Distal Third Nonunion, additionally, (c) Multiple distal interlocking screws in different planes in distal fragment were added to conform additional stability. (d) Blocking screws.
By definition, isthmus of femur was defined as the narrowest region between the cortices of femur shaft on AP view. Distal Third region was defined as region between the isthmus and the upper border of trans-epicondylar width in knee joint [5].

\section{Technique}

All patients were positioned in supine position on the radiolucent fracture table. All nails were exchanged in anterograde manner. After nail removal, the intramedullary canal was sequentially over-reamed until strong resistance was felt or bony debris were seen within the reamer flutes. The increased nail diameter was exchanged in an anterograde manner with nail diameter at least 2 $\mathrm{mm}$ larger than the previous nail. The mean nail diameter of nail inserted in primary procedure was $9.45 \mathrm{~mm}$ (range 8 - 12) whereas

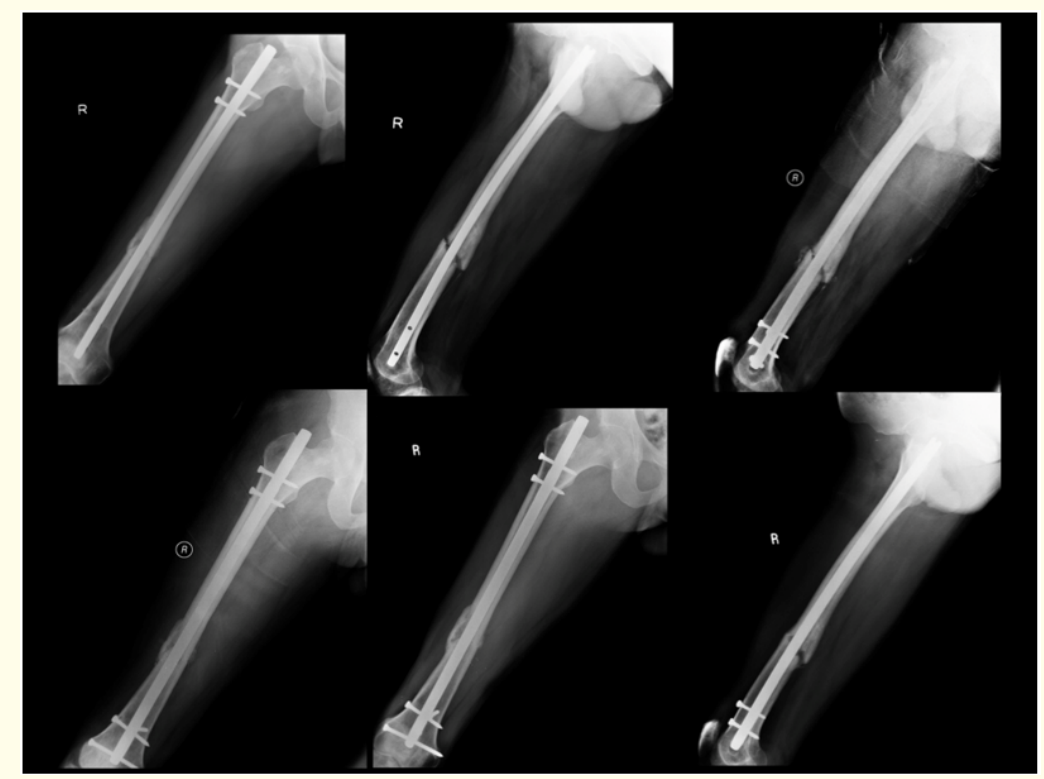

Figure 3 40-year-old male presenting with Distal Third non-union with intramedullary nail in situ at 12 months.

Exchange nailing with poller screw was done and union was achieved at 12 months post exchange nailing.

the mean exchanged nail diameter was $11.20 \mathrm{~mm}$ (range 10 - 14). Furthermore, poller screws were inserted in the distal fragment of Distal Third non-union in all cases of exchange nailing. Additional Interlocking screws were added in distal fragment in multiplanar directions. The poller screw was positioned in the sagittal plane of the distal fragment closest to the nail. All the screws were selftapping, predrilled screws inserted using drill guides. Additionally, all the nails were reimpacted ("backslapped") after distal locking to improve contact at non-union site.

All patients received physiotherapy postoperatively. Weight bearing was allowed as tolerated and follow up X-rays were taken serially at 6-week intervals. Clinical assessment of weight bearing, wound healing and functionality were assessed at 3 months postoperatively (Figure 2).

Non continuous variables were tested using Fisher Exact Test. Main outcome measures (radiological union and clinical union) were evaluated using multiple logistic regression analysis. One way analysis of variance was used to determine time to healing and nail diameter. Criteria for significance was $\mathrm{p}<0.05$.

\section{Results}

Out of 55 patients, 5 were lost to follow up. Remaining 50 were analysed and 47 (94\%) patients went on to heal radiologically at a mean of 8.52 (range 3 - 18 months). Remaining 3 patients refused 
further treatment (Table 1).

25 isthmus nonunion healed out of total $29(86.2 \%)$ at a mean duration of 7.60 months. Of the remaining 4, 3 refused further surgery and 1 was treated surgically with bone grafting and went on to heal at 18 months postoperatively. of the 25 isthmus non-union that healed successfully, 3 required dynamisation and went on to heal at a mean of 13 months. 1 patient had superficial infection which resolved with debridement at 4 months. Harris Hip Score

1. Taitsman LA., et al. "Risk factors for femoral nonunion after femoral shaft fracture". The Journal of Trauma 67.6 (2009): $1389-1392$.

2. Canadian Orthopaedic Trauma Society. "Nonunion following intramedullary nailing of the femur with and without reaming. Results of a multicenter randomized clinical trial". The Journal of Bone and Joint Surgery American Volume 85.11 (2003): 2093-2096.

3. Wolinsky PR., et al. "Reamed intramedullary nailing of the femur: 551 cases". The Journal of Trauma 46.3 (1999): 392-399.

4. Zickel RE. "Nonunions of fractures of the proximal and distal thirds of the shaft of the femur". Instructional Course Lectures 37 (1988): 173-179.

5. Yang KH., et al. "Nonisthmus femoral shaft nonunion as a risk factor for exchange nailing failure". The Journal of Trauma and Acute Care Surgery 72.2 (2012): E60-E64.

6. Pihlajamaki HK., et al. "The treatment of nonunions following intramedullary nailing of femoral shaft fractures". Journal of Orthopaedic Trauma 16.6 (2002): 394-402.

7. Shroeder JE., et al. "The outcome of closed, intramedullary exchange nailing with reamed insertion in the treatment of femoral shaft nonunions". Journal of Orthopaedic Trauma 23.9 (2009): 653-657.

8. Danckwardt-Lilliestrom G. "Reaming of the medullary cavity and its effect on diaphyseal bone. A fluorochromic, microangiographic and histologic study on the rabbit tibia and dog femur". Acta Orthopaedica Scandinavica Supplementum 128 (1969): 1-153.

9. Grundnes 0 and Reikeras O. "Acute effects of intramedullary reaming on bone blood flow in rats". Acta Orthopaedica Scandinavica 64.2 (1993): 203-206.
10. Reichert IL., et al. "The acute vascular response to intramedullary reaming. Microsphere estimation of blood flow in the intact ovine tibia". The Journal of Bone and Joint Surgery British Volume 77.3 (1995): 490-493.

11. Russell TA., et al. "Mechanical characterization of femoral interlocking intramedullary nailing systems". Journal of Orthopaedic Trauma 5.3 (1991): 332-340.

12. Utvag SE., et al. "Graded exchange reaming and nailing of nonunions. Strength and mineralisation in rat femoral bone". Archives of Orthopaedic and Trauma Surgery 118.1-2 (1998): 1-6.

13. Weresh MJ., et al. "Failure of exchange reamed intramedullary nails for ununited femoral shaft fractures". Journal of Orthopaedic Trauma 14.5 (2000): 335-338.

14. Banaszkiewicz PA., et al. "Femoral exchange nailing for aseptic non-union: not the end to all problems". Injury 34.5 (2003): 349-356.

15. Cleveland KB. "Delayed union and nonunion of fractures". In Canale and Beaty: Campbell's Operative Orthopaedics, Volume III. $12^{\text {th }}$ edition. Edited by Canale ST, Beaty JH. Philadelphia: Mosby, Chapter 59 (2013).

16. Heckman JD., et al. "Acceleration of tibial fracture-healing by non-invasive, low-intensity pulsed ultrasound". The Journal of Bone and Joint Surgery American Volume 76.1 (1994): 26-34.

17. Kessler SB., et al. "The effects of reaming and intramedullary nailing on fracture healing". Clinical Orthopaedics and Related Research 212 (1986): 18-25.

18. Brumback RJ. "The rationales of interlocking nailing of the femur, tibia, and humerus". Clinical Orthopaedics and Related Research 324 (1996): 292-320.

19. Niedzwiedzki T., et al. "Treatment of femoral shaft union disturbances with intramedullary nailing. Treatment failure". Ortopedia, Traumatologia, Rehabilitacja 9.4 (2007): 377-383.

20. Brinker MR and O'Connor DP. "Nonunions: evaluation and treatment". In: Browner BD, Jupiter JB, Levine AM., et al. eds. Skeletal Trauma: Basic Science, Management, and Reconstruction. Philadelphia, PA: W.B. Saunders (2009): 615-707.

21. Frolke JP., et al. "Viable osteoblastic potential of cortical reamings from intramedullary nailing". Journal of Orthopaedic Research 22.6 (2004): 1271-1275.

22. Wenisch S., et al. "Human reaming debris: a source of multipotent stem cells". Bone 36.1 (2005): 74-83. 
23. Gelalis ID., et al. "Diagnostic and treatment modalities in nonunions of the femoral shaft: a review". Injury 43.7 (2012): 980-988.

24. Zhang X., et al. "Treatment of distal femoral nonunion and delayed union by using a retrograde intramedullary interlocking nail". Chinese Journal of Traumatology = Zhonghua Chuang Shang Za Zhi 4.3 (2001): 180-184.

25. Ueng SW., et al. "Augmentative plate fixation for the management of femoral nonunion after intramedullary nailing". The Journal of Trauma 43.4 (1997): 640-644.

26. Krettek C., et al. "The mechanical effect of blocking screws ("Poller screws") in stabilizing tibia fractures with short proximal or distal fragments after insertion of small-diameter intramedullary nails". Journal of Orthopaedic Trauma 13.8 (1999): 550-553.

\section{Assets from publication with us}

- Prompt Acknowledgement after receiving the article

- Thorough Double blinded peer review

- Rapid Publication

- Issue of Publication Certificate

- High visibility of your Published work

Website: https://www.actascientific.com/

Submit Article: https://www.actascientific.com/submission.php Email us: editor@actascientific.com

Contact us: +919182824667 